\title{
Éditorial \\ Apprendre une langue des signes
}

\author{
Christian Cuxac \\ Université Paris-VIII
}

Cet article est une version remaniée d'une publication antérieure, parue en 2001 et intitulée « apprendre la LSF », qui s'adressait aux personnes entendantes - plus particulièrement aux parents entendants d'enfants sourds-, désireuses de se lancer dans l'apprentissage de la langue des signes française (LSF) ${ }^{1}$. La grande ressemblance des langues des signes entre elles quant à leur organisation linguistique nous a autorisé à étendre les remarques qui s'appliquaient initialement à la LSF à l'ensemble des langues des signes (LS) du monde, reprenant en cela la belle métaphore formulée en $1981^{2}$ par Joe Castronovo, poète sourd américain:

Le langage, c'est comme la mer. Les langues individuelles, soit les langues des signes, soit les langues vocales, sont les fleuves qui vont à la mer. Il y a des ressemblances fondamentales entre langues des signes et langues vocales. Mais il y a aussi des différences très importantes entre signeurs sourds et locuteurs entendants, et leur rapport avec le langage et leurs langues. Les locuteurs entendants, les pauvres, ne savent pas bien nager dans la mer du langage, ni dans les fleuves de leurs langues vocales. Ainsi, s'ils veulent aller de leur langue à une autre langue, ils sont forcés de construire des ponts, et puis de marcher sur ces ponts pour voyager d'une langue à l'autre. Par contre, les signeurs sourds sont de très bons nageurs qui n'ont pas peur de plonger, ni dans la mer du langage, ni dans les fleuves de leurs langues des signes. Ainsi, ils n'ont pas besoin de construire des ponts pour se promener de l'une à l'autre de leurs langues des signes mais, tout simplement, ils nagent, tranquilles, soit dans les fleuves de leurs langues, soit dans la mer du langage.

Imaginons que vous soyez entendant et que vous veniez de prendre la décision d'apprendre une langue des signes. Quelles qu'en soient les raisons et les modalités d'accès (cours formels hebdomadaires ou cours intensifs dans des associations ou à l'université, contact direct avec des sourds), les débutants ont sur les apprenants avancés l'avantage incontestable d'être en phase de découverte.

Vous étiez monolingue (ou bilingue, ou trilingue) et bientôt vous allez pratiquer une langue de pus. Quoi de plus excitant et de plus enviable aussi : il vaut toujours mieux connaître deux langues qu'une, trois que deux, etc. Et quoi de plus positif et de plus gratifiant pour vous : bientôt, vous allez pouvoir discuter avec des locuteurs sourds natifs

\footnotetext{
${ }^{1}$ Christian Cuxac, 2001, Apprendre la LSF, in Surdité et souffrance psychique, ed. Frédéric Peillon, Ellipses, Paris, pp. 35 - 41

${ }^{2}$ Communication personnelle à Elena Antinoro Pizzuto, en ASL, à l'occasion du spectacle conçu par Joe Castronovo et Ignazio Buttitta "Yeux - Poésie en langue des signes et posie en dialecte se rencontrent », Palerme, Librerie Astersco, mai 1981 (annoté et traduit par Elena Antinoro Pizzuto.
} 
dans leur langue, une langue qui n'est pas la vôtre. Vous avez fait le geste positif d'aller vers l'autre et celui-ci ne peut que vous en savoir gré. Une aventure intellectuelle aussi commence, puisque vous allez apprendre à découper le monde différemment à dire et à vous comporter culturellement d'une autre manière.

\section{De nouveaux comportements}

En outre, ce plaisir à pratiquer une autre langue, et à s'ouvrir à une autre façon de voir et de dire, est d'autant plus vif que l'objet linguistique à atteindre est plus éloigné de vos cadres premiers de référence. Et, sur ce plan, l'écart entre vos habitudes linguistiques et celles qui impliquent la pratique d'une langue des signes est, sur de nombreux points, maximal.

a) Ne plus parler

Vous aurez ainsi la surprise de discourir, d'échanger des informations en situation de face à face en silence, sans avoir à donner de la voix. Certes, parler en même temps que vous faites des gestes est possible (des phrases qui suivent la grammaire de votre langue vocale en y ajoutant des gestes de la langue des signes), mais c'est une habitude à ne pas prendre. C'est un peu comme si, n'étant ni dans une langue ni dans l'autre et en mélangeant anglais et français, un débutant vous disait «Comment faire vous faîtes? (anglais « How do you do ?») pour vous demander «Comment ça va ?».

b) Construire du sens avec son corps

Apprendre une langue des signes, c'est aussi apprendre à utiliser d'une autre manière son corps, tout mouvement gestuel et facial devenant chargé de sens, une autre façon d'utiliser son regard aussi. En tant qu'émetteur du message, vous saurez que le regard est l'élément qui commande l'interaction et a, de ce fait, valeur de « je » et de «tu ». Et que, si vous êtes le récepteur du message, celui à qui l'on s'adresse, il est nécessaire de regarder en permanence en vision centrale le visage de votre interlocuteur. Vous abandonnerez alors la tentation de suivre les mouvements des gestes de votre interlocuteur, auquel cas vous vous condamneriez à ne rien comprendre de ce qu'il vous dit. On reconnaît d'ailleurs tout de suite un apprenant débutant à ce comportement.

C'est donc toute une nouvelle éducation du système visuel qui, pour vous, commence, en raison du changement de canal. Vous serez ainsi surpris par l'impression de (saine) fatigue physique et intellectuelle qui suit les premiers cours, faute d'habitude, dans le monde des entendants, de décoder des messages complexes à la fois temporels et spatiaux où plusieurs informations en mouvement sont délivrées en même temps. 


\section{Premières découvertes linguistiques}

Assez vite, vous vous apercevrez qu'il y a comme deux langues des signes : une langue des signes qui dit en donnant à voir, en figurant, en illustrant; et une langue des signes qui, comme les langues vocales, dit sans donner à voir.

a) Une langue qui peut donner à voir en disant

Premier aspect des langues des signes, lorsque le locuteur dit en montrant, en donnant à voir, vous le découvrirez lorsque votre interlocuteur sourd vous racontera des histoires drôles, ou bien le récit de ses dernières vacances, ou bien celui du film quil vient de voir. Le principe qui régit les structures -elles sont au nombre de trois- qui permettent de montrer est assez simple. Il consiste en : 1) soit une figuration manuelle des formes ou des contours de forme d'un objet, d'un animal, d'un personnage humain ; 2) soit la figuration au moyen des deux mains du déplacement d'un personnage ou d'un objet comme vu de loin et en mouvement par rapport à un lieu fixe ; soit une figuration consistant à « devenir » un personnage humain ou animal, voire même un objet, au moyen d'une sorte d'incorporation que les sourds appellent « rôle» ou «prise de rôle ». Ces deux derniers types de figuration peuvent être combinés simultanément.

Le plus étonnant est que, lorsque les sourds construisent des récits très figuratifs, tout, sans être transparent, paraît plus clair, presque évident -l'impression que «on ne pourrait pas mieux dire »- même pour un entendant qui débute en langue des signes. On a souvent caractérisé ce productions comme relevant du mime ou de la pantomime, mais à tort : les recherches récentes consacrées aux LS montrent bien à quel point ces messages sont finement structurés et en tous points redevables d'analyses linguistiques. La preuve en est fournie par le paradoxe suivant : tout aussi facilement décodables qu'elles soient, de telles séquences sont extrêmement délicates à réaliser par les entendants ; ce sont aussi les plus tardives et les plus difficiles à acquérir. Pour faire comprendre de quelles constructions il s'agit, je pense qu'il conviendrait de ne pas se référer aux narrations auxquelles nous sommes habitués dans nos langues vocales et écrites, mais plutôt aux techniques narratives cinématographiques (changements de plans, éclairage d'une scène à partir d'angles de prise de vue différents), bref, de tout ce qui s'élabore lors de la mise en scène, puis au montage.

b) Une utilisation massive de l'espace 
Parallèlement à cela, se poursuit l'apprentissage de l'autre versant de la langue des signes, lorsqu'elle dit sans donner à voir, sans illustrer, avec son vocabulaire de plusieurs milliers de signes qui seront acquis peu à peu, ainsi que les principes d'une grammaire qui utilise essentiellement l'espace. Vous apprendrez donc à déplacer certains signes dans l'espace et, pour ceux qui ne peuvent être déplacés, parce qu'ils sont réalisés sur un point fixe du corps (par exemple, en LSF «garçon » au niveau du front, « fille » au niveau de la joue), à les reprendre au moyen d'un pointage de l'index et, grâce à la direction de ce pointage, à leur attribuer une place particulière, comme une sorte de mise en mémoire spatiale (à droite ou à gauche, devant soi, plus haut ou plus bas, etc.). Ce placement dans l'espace par pointage est capital pour savoir qui fait quoi ; en effet, une bonne partie des verbes des LS vont relier ces différents pointages (et donc les signes qui leur correspondent et qu'ils reprennent) grâce au point de départ et d'arrivée de leur mouvement. Cela permet de savoir si c'est le garçon qui invite, informe, répond à, dit à, etc., la fille, ou bien l'inverse. Vous apprendrez aussi à spatialiser le temps : en LSF par exemple, selon une ligne de temps allant de l'arrière du corps (le passé) à l'avant (le futur) ${ }^{3}$ lorsque ce que vous dites est en relation avec le temps présent (par exemple lorsque vous dites « dans trois ans », « hier », «plus tard ») ; et, lorsque ce n'est pas le cas, à placer une date de référence en un point de l'espace (toujours comme une sorte de mise en mémoire spatiale) date par rapport à laquelle ce qui s'est passé avant ou ce qui se passera après s'indique selon une ligne de temps horizontale. Les verbes, alors, pourront être déplacés le long de cette ligne de temps et, en fonction de leur place, se chargeront de valeurs temporelles différentes.

\section{Le corps, comme un orchestre}

Pour faire sens, l'ensemble du corps est utilisé, et pas seulement les mains.

a) Le rôle du regard

On a déjà dit que le regard permettait d'entrer en interaction, mais le regard active aussi des portions d'espace où les gestes vont venir s'installer (un peu comme le fait la souris de l'ordinateur qui active l'écran à tel endroit précis). Parfois, aussi, il convient de porter le regard sur certains des signes que l'on est soi-même en train de réaliser, faute de quoi, le message n'aurait pas forcément de sens.

b) La mimique faciale

\footnotetext{
${ }^{3}$ Ce fléchage spatial du temps est universel, présent aussi bien dans les LS que dans la gestuelle co verbale des entendants. En revanche, ce qui n'est pas universel est la direction de ce fléchage de par le monde : certaines populations entendantes placent par exemple, dans leur gestualité co verbale, le futur derrière le corps et le passé devant.
} 
On découvrira aussi que les expressions du visage, ou mimique, ont une importance considérable : lorsque la langue des signes donne à voir, réalisée en même temps que les formes effectuées par les mains, c'est la mimique faciale qui permet de qualifier ces formes comme belles, moches, petites, grosses, moyennes, spongieuses, etc. Lorsque la langue des signes dit sans donner à voir, la mimique permet de quantifier les noms (beaucoup de, un peu de). Accompagnant les verbes elle indique la façon dont s'accomplit l'action : avec attention, avec effort, facilement, rapidement, etc. Enfin, appliquée à la phrase entière, la mimique fait savoir mon opinion sur ce que je suis en train de dire : si c'est une question, une condition, une mise en doute, une assertion, etc.

c) Mouvements du corps et du visage

Par ailleurs, les balancements du corps permettent de relier des phrases entre elles et prennent la valeur de « et » ou de «ou bien », et de minuscules hochements de tête ont pour rôle d'assurer le suivi et le maintien de l'interaction («Tu me suis ? , «N'est-ce pas ? , «Hein?», etc.). Pour résumer, le corps du locuteur en langue des signes pourrait faire penser à un orchestre symphonique dont les différentes parties s'harmonisent les unes par rapport aux autres.

\section{En guise de conclusion}

Comme pour toute langue, il vous faudra du temps pour apprendre une langue des signes. Vous aurez parfois l'impression de ne pas avancer, de ne pas faire de progrès notables, et parfois, au contraire, vous aurez la sensation mystérieuse d'avoir franchi subitement un cap important. Et puis, un jour, vous constaterez, au hasard d'une rencontre avec des sourds que vous ne connaissiez pas, que vous pouvez communiquer sans problème avec eux. A plus forte raison, lorsque, rencontrant des sourds étrangers et échangeant sans problème avec eux, vous serez amené à constater que la pratique fluide d'une langue des signes ouvre à une communication planétaire, la pratique de l'une d'elles vous permettant d'entrer en contact rapidement et efficacement en relation linguistique avec quiconque en pratique une autre. Enfin, à ce stade avancé, vous constaterez qu'en parallèle à l'apprentissage d'une langue des signes, vous aurez appris à discipliner votre corps et votre gestuelle, à éduquer votre regard et à en accroître les performances, et à produire avec votre visage un nombre d'expressions faciales que vous n'auriez jamais soupçonné pouvoir réaliser. J'ai pris le risque ici-même de déflorer ce que vous auriez eu la surprise de découvrir par vous-même au long de votre apprentissage d'une langue des signes, et de rendre explicites certains aspects de cette langue que vous n'auriez pas eu nécessairement 
conscience d'avoir acquis. En ce sens, c'est un peu dommage, et je le regrette ; mais si cet article, en aiguisant leur curiosité pouvait inciter certains à faire le pas d'apprendre une langue des signes, l'effet dommageable de cette anticipation en serait largement compensé. 